\title{
A Novel Run-length based wavelet features for Screening Thyroid Nodule Malignancy
}

\author{
Salih Omer Haji ${ }^{\star}$ \\ https://orcid.org/0000-0002-6291-6423 \\ Raghad Zuhair Yousif ${ }^{1}$ \\ https://orcid.org/0000-0002-5683-9695 \\ ${ }^{1}$ Salahaddin University, College of Science, Physics Department, Erbil, Iraq; \\ Received: 2017.12.30; Accepted: 2019.06.06.
}

*Correspondence: salih.haji@su.edu.krd; Tel.: +9647504514643 (S.O.H)

\section{HIGHLIGHTS}

- An automatic and intelligent system were proposed to screen thyroid nodule cancer.

- A hybrid features (with and without wavelet) transform were derived.

- 33 out of 45 features passed Anova test based on run length statistical features.

- SVM classifier outperformers other classifiers with an average accuracy of $97 \%$.

\begin{abstract}
Thyroid nodules are cell growths in the thyroid which might be for in one of two categories benign or malignant. Nodular thyroid disease is common and because of the associated risk of malignancy and hyper-function; these nodules have to be examined thoroughly. Hence diagnosing thyroid nodule malignancy in the early stage can mitigate the possibility of death. This paper presents an intelligent thyroid nodules malignancy diagnosis using texture information in run-length matrix derived from 2level 2D wavelet transform bands (approximation and details). In this work, ANOVA test has been used to for feature selection to reduce for feature selection about 45 run-length features with and without wavelet generated, before feeding those features which clinical importance to the Support Vector Machine(SVM) and Decision Tree (DT) classifier to perform the automated diagnosis. The validation of this work is activated using 100-thyroid nodule images spliced equally between the two categories (50 Benign and 50 Malignant). The proposed system can detect thyroid nodules malignancy with an average accuracy of about $97 \%$ using SVM classifier for the runlength matrix, features derived from spatial domain while the average accuracy is increased to $98 \%$ in case of hybrid feature derived from spatial domain and 2-level wavelet decomposition. For the other proposed classifier (DT), the average accuracy
\end{abstract}


in case of spatial domain based features is $93 \%$ whereas the average accuracy of the hybrid features system is $97 \%$. Hence the proposed system can be used for the screening of thyroid nodules.

Keywords: Thyroid Nodule; SVM and DT classifiers; computer-aided iagnosis; feature extraction; Run-Length Matrix.

\section{INTRODUCTION}

The thyroid organ is found low in the front of the neck. The organ is formed like a butterfly and wraps around the windpipe or trachea. The two wings on either side of the windpipe are joined together by a bridge, called the isthmus, which traverses the front of the windpipe as shown in Figure 1.

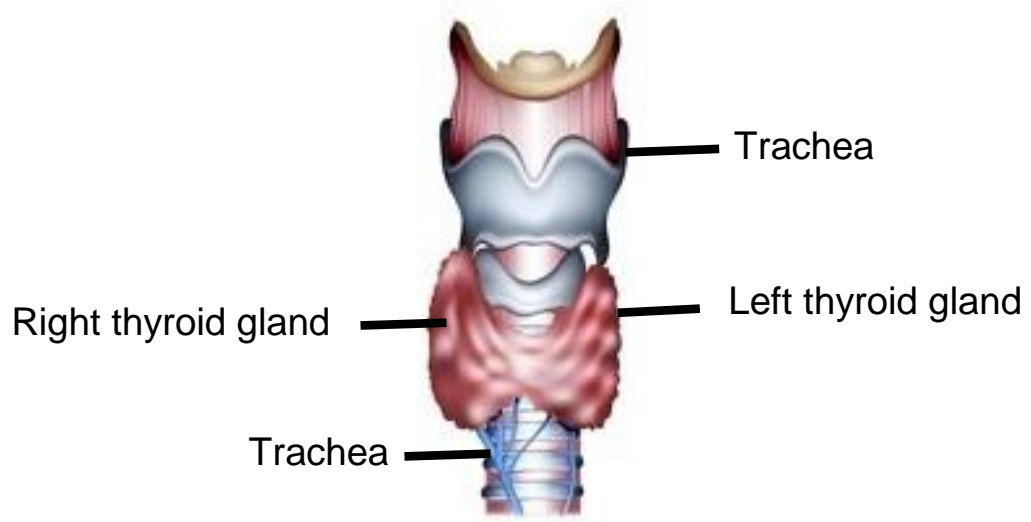

Figure 1. Thyroid gland.

According to the World Health Organization (WHO), cancer is one of the main sources of death overall causing 7.6 million deaths in 2008 and 8.2 million deaths in 2012. Yearly growth mortalities are required to ascend to 22 million inside the following two decades. In 2018, new tumor instances of 1,735,350 and malignancy deaths of 609,640 occurred in the Assembled States alone [1]. Thyroid cancer is the seventh most regular malignancy in ladies and fifteenth most basic growth in men. Also, the death rates increase with age. The main causes of thyroid cancer are high radiation dosage, family history, aging, low iodine diet, familial adenomatous polyposis, Cowden disease and carney complex [2]. Thyroid nodule, one of the regular issue of the organ, can be strong mass, cysts which are either benign (non- cancerous) or malignant (cancerous) [3]. Thyroid cancer commences from an atypical growth of thyroid tissue at the edge of the thyroid gland. Initially, it forms a lump in the throat and an over-growth of this tissue leads to the formation of benign or malignant thyroid nodules. Nodular thyroid disease is common because of the associated risk of malignancy and hyperfunction; these nodules have to be examined thoroughly. A Fine Needle Aspiration Biopsy (FNAB) is a non-surgical and acknowledged symptomatic technique for affirming the idea of the nodules. In any case, it prompts (10-20) \% thyroid nodules with no diagnostic, however, (15-30) \% of results are uncertain. (FNAB) is a non-surgical and an accepted method for confirming the nature of the nodules. Thyroid nodules have a high probability of malignancy and aspiration should be repeated [4,5]. The risk of developing a palpable thyroid nodule in a lifetime ranges between (5-10) \% [6]. Numerous modalities like ultrasonography, radiographs, scintigraphy, Computed Tomography (CT), and Magnetic Resonance Imaging (MRI) are accessible for thyroid nodule imaging [7]. Among them, the most favored decision is thyroid ultrasonography 
which is a non-obtrusive indicative test that gives an image of the structure and the attributes of thyroid nodules. Figure 2 demonstrates normal thyroid Ultrasound (US) images for the two classes (Benign and Malignant).

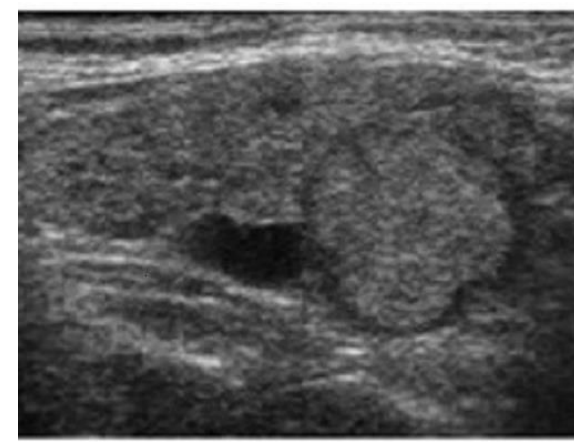

(a)

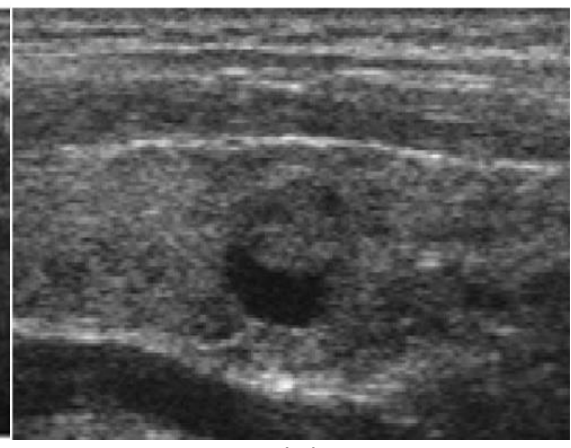

(b)

Figure 2. Typical Thyroid US Images: (a) Benign (b) Malignant.

Ultrasound-based computer-aided diagnosis (CAD) for tumor diseases provides an effective decision support and second opinion tool for radiologists. Manual and semiautomated diagnosis of thyroid nodules is dull tedious and may cause observer inconstancy errors while satisfying the structural abnormalities of the nodules by various clinicians. The symptomatic result may get influenced regardless of the possibility that there is an error in the division. Along these lines, (CAD) can help the clinicians to determine these problems and can be utilized as assistant devices by the clinicians to cross-check their diagnosis $[8,9]$. These CAD tools analyze the entire input image and extract the salient features. CAD techniques do not depend on the individual segmentation and measurement of the various geometric parameters in order to even a small error in the segmentation may lead to wrong diagnosis [10]. The CAD strategies are quick, economical and can be executed even without a specialist's help.

Feature representation plays an essential role in ultrasound-based CAD [11]. Texture information is among the most commonly used features in ultrasound image 12. Numerous texture feature extraction methods, depending on wavelet transform, and other statistical descriptors [11-14], have been used for ultrasound images. Jenitta and Samson [15] utilized Local Vector Pattern (LVP) to separate fine features present in the geographical image and recover the pertinent images from a vast remote sensing image database.

Xiaoon Tang [16] enhances image grouping precision by utilizing customary runlength system and show that the run-length frameworks contain awesome oppressive data and that a decent strategy for extricating such data is of central significance to fruitful order. Conners, R.W. also, C.A. Conners, R.W. and C.A. Harlow [17], have found that these run length related features have not performed as well as other features such as spatial gray level dependence and gray level difference. This shows a clear need for a more explicit gray level distribution related information within the texture descriptive features. Presumably with a view to addressing this need, Chu, Sehgal and Greenleaf [18] proposed a couple of new features that reflected the gray level distribution with regards to the run length grid. On the other hand, Bhuvaneswari and Sivakumar [19] utilized a novel image classification procedure that utilized the particle filter framework (PFF)-based improvement strategy for satellite image classification. While Reena Rose et al. [20] utilizes (SVM) to delineate contrasts in performance with respect to arranged issues in face recognition using six benchmark databases.

In this paper, a classification of uncertain lesion has been investigated using features derived from run-length metrics with a new approach, involves calculating the 
two-level decomposition wavelet coefficients, then quantize these coefficients taken from four decomposition bands before calculating the run-length matrix. A hybrid technique is proposed which includes extracting texture run-length features directly from spatial domain and from wavelet transform. The hybrid technique overcomes a limitation that can be arises by utilizing single scale analysis (depend on spatial domain data) [21]. In view of the presumption that texture can be totally characterized from the statistical properties of its multi-scale representation, Wavelet coefficients' first and second order statistics have been proposed [22]. Wavelet Transform has an advantage for pattern recognition. With wavelet transform it is able to isolate the signal as much as it can, at first it has brought the signal through with low pass and then high pass filters. With this arrangement, it might isolate two signals with sub-band divisions.

\section{MATERIAL AND METHODS}

The flow chart of the proposed system is illustrated in Figure 3, composed of four main stages. The first stage is image pre-processing, while the second stage is feature extraction in which more than 45 statistical features have been generated from each input image to this stage. The main two sources of preprocessed image features are Gray Level Run-Length Matrix (GLRLM), and 1st and 2nd levels wavelet decomposition based GLRLM. The third stage involves a feature selection procedure using ANOVA with (P-value $<0.001$ ). The final stage relies upon specialists' reports about various classes to which the thyroid nodules belongs. Moreover, the extracted significant features set are passed to the classifier. Two types of classifier have been utilized (SVM and DT). The classifier performance is evaluated using ten-fold cross validation strategy. This paper consists of the following sections: The images utilized for this study, pre-processing, wavelet and wavelet coefficient quantization, feature extraction and feature selection illustrated in section 2, and the classifiers utilized for diagnosis, SVM and DT, are explained in section 3. The result evaluated is depicted in section 4. Finally, the conclusion declared in section 5. 


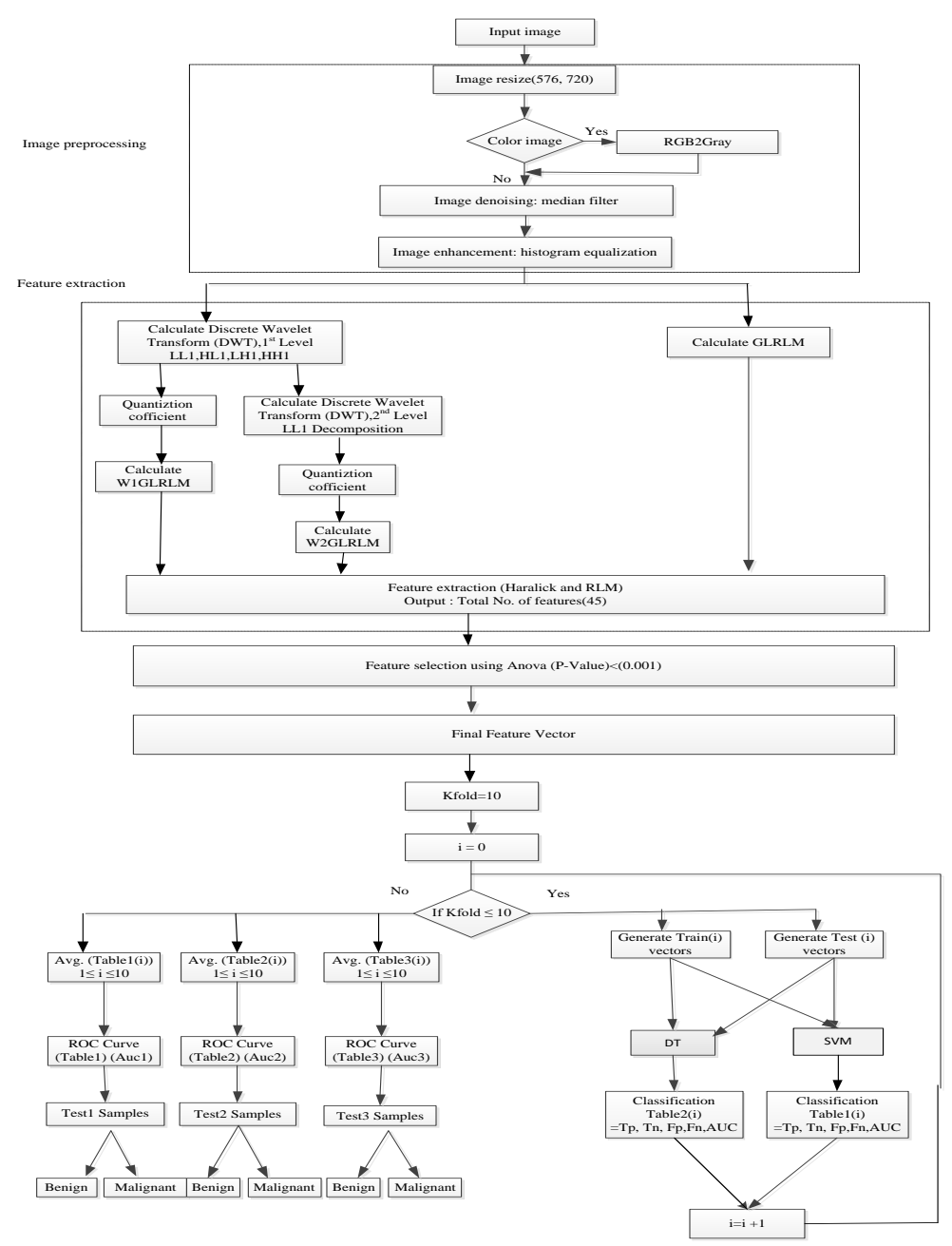

Figure 3. Proposed system.

\section{Data acquisition}

The data used in this study consisted of 100 fine-needle aspiration biopsy-proven lesions (50 benign and 50 malignant) were collected in JPEG format within the various age groups and different sizes (rows $\times$ columns). The images were extracted from thyroid ultrasound video sequences captured with a TOSHIBA Nemio30 and a TOSHIBA Nemio MX Ultrasound devices, both set to $12 \mathrm{MHz}$ convex and linear transducers. Thyroid nodules images saved in ultrasonography system that includes a complete annotation and diagnostic description of suspicious thyroid lesions, using the TI-RADS lexicon description performed by at least two expert radiologists. An arrangement of cases with significant thyroid issue was chosen from the IDIME Ultrasound Division (Foundation with more than ten years of experience), one of the biggest indicative imaging focuses in Colombia and who performs more than 2000 thyroid ultrasounds related to Fine Needle Aspiration every year. The choice of patients depended on TI-RADS description [23].

\section{Preprocessing of image data}

Feature extraction is most vital part in this study and is broadly utilized as a part of classification processes. This extraction is completed after preprocessing the images. The acquired thyroid images were very high different resolutions. To process these images directly takes a lot of processing time. Hence to reduce the computation time we resize the thyroid nodule images into $576 \times 720$. Figure 4 , 
demonstrates the pre- preparing step fundamentally comprises of resizing image to 576 by 720 , at that point acquainted with the Median filter which is a non-linear filter type and used to reduce the effect of noise without blurring the sharp edge. The operation of the Median filter is - first organize the pixel esteems in either the ascending or descending order and then compute the median value of the neighborhood. Additionally, Adaptive Histogram Equalization (AHE) which is a computer image processing technique used to improve contrast in.

Image improvement is an essential stride in preprocessing stage. The image enhancement is to build the visual impression of the image that they are more reasonable for human watchers or machine vision applications. It is notable in the image preparing society that there is no bringing together or general hypothesis for image upgrade calculations. Along these lines, an enhancement algorithm that is reasonable for some application may not work in different applications. In image processing technology, image enhancement means improving image quality through a broad range of techniques such as contrast enhancement, color enhancement, dynamic range expansion, edge emphasis, et cetera.

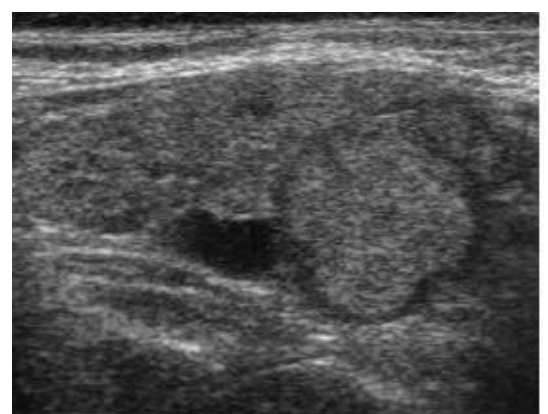

a. Original Thyroid nodules image

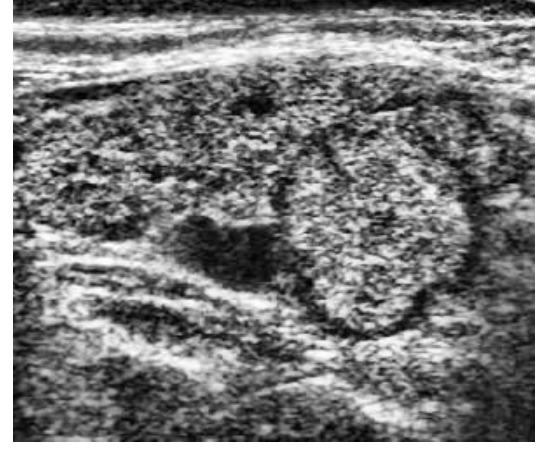

c. Median filtered image

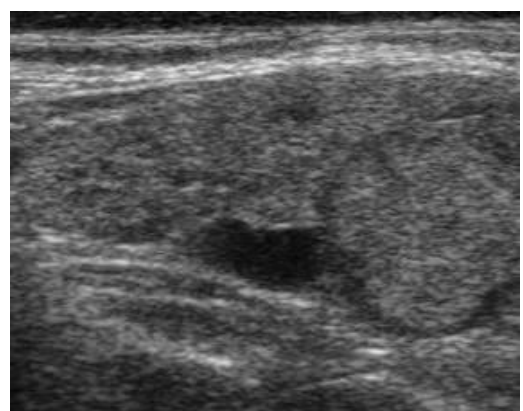

b. Resized Thyroid nodules image to $576 * 720$ pixel

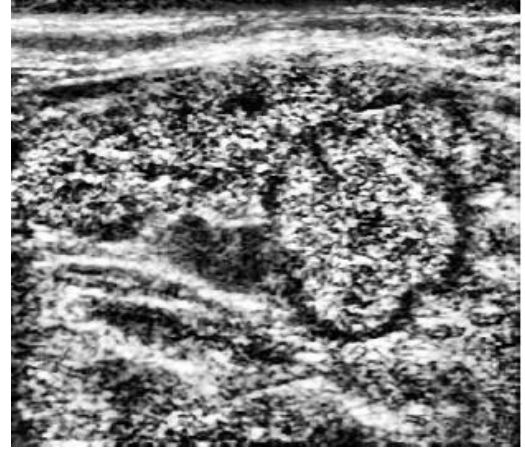

d. Adaptive histogram equalization image

Figure 4. Image pre-processing steps.

\section{Wavelet}

Wavelet transforms the image from space domain to local frequency domain. It involves a group of low pass and high pass filters named filter bands. The Discreet Wavelet Transform(DWT) uses filter bands to carryout wavelet analysis and divides the image into various frequency bands. The wavelet transform converts the image into multi-scale representation to get both of spatial and the frequency characteristics. This leads to efficient multi-scale image analysis with lower computational cost [24]. It generates orientation sensitive data which very important in analyzing thyroid nodules.

The DWT-2D corresponds to multi-resolution approximation expressions [25]. 


$$
f(x, y)=\sum_{j=0}^{N_{J}-1} S_{j} \varphi_{j n}(x, y)+\sum_{j=0}^{N_{J}-1} \sum_{k=0}^{J} d_{j k} \psi_{j k}(x, y)
$$

where $\mathrm{J}$ represents the scale of the analysis, and scale $\mathrm{j}$ indicates the coarsest scale or lowest resolution of the analysis, $\mathrm{N}_{\mathrm{J}}=\mathrm{N} / 2 \mathrm{j}$, is the number of coefficients in scale $\mathrm{j}$. As shown in equation (2) the procedure of wavelet decomposition is as follows: wavelet has two functions "wavelet" and "scaling function". They are such that there are half the frequencies between them. They act as a low pass filter and a high pass filter, $\varphi_{n}$ is the scale function which can be defined by:

$$
\varphi_{n}=\varphi(t-n) \quad n \in Z, \varphi \in L^{2}
$$

The subspace of $L 2(R)$ spanned by these functions is defined as:

$$
V_{0}=\overline{\operatorname{span}\left\{\varphi_{n}(t)\right\}}
$$

This means that:

$$
f(t)=\sum_{n} S_{n} \varphi_{n}(t) \ldots \ldots . . \forall . . f(t) \in V_{0}
$$

One can generally increase the size of the subspace spanned by changing the time scale of the scaling functions. A two-dimensional family of functions is generated from the basic scaling function by scaling and translation as illustrated below:

$$
\varphi_{j, n}(t)=2^{j / 2} \varphi\left(2^{j} t-n\right)
$$

Whose span over $k$ is:

$$
V_{j}=\overline{\operatorname{span}\left\{\varphi_{j, n}(t)\right\}}
$$

If $f(t) \in V_{j}$, it can be expressed as:

$$
f(t)=\sum_{n} S_{n} \varphi\left(2^{j} t+n\right)
$$

If $\varphi(t)$ is in $V 0$ it is also in $V 1$, this mean that $\varphi(t)$ can be expressed in terms of a weighted sum of shifted $\varphi(2 t)$ as:

$$
\varphi(t)=\sum_{n} h(n) \sqrt{2} \varphi(2 t-n) \quad n \in Z
$$

Where $h(n)$ is a sequence of real (or complex) numbers called the scaling function coefficients (or the scaling filter). In addition to the scaling function $\varphi n$ that has been discussed, another basic function called the wavelet function $\psi_{\mathrm{k}}$ is required. Dilation and translations of the "Mother function", or "analyzing wavelet" $\psi(x)$, define an orthogonal basis, our wavelet basis:

$$
\psi_{(s, l)}=2^{\frac{-s}{2}} \psi\left(2^{-s} x-l\right)
$$

The variables $s$ and $I$ are integers that scale and dilate the mother function to generate wavelets. The scale index $s$ indicates the wavelet's width, and the location 
index I gives its position. To span our data domain at different resolutions, the analyzing wavelet is used in a scaling equation:

$$
W(x)=\sum_{k=-1}^{N-2}(-1)^{k} C_{k+1} \psi(2 x+k)
$$

Where $W(x)$ is the scaling function for the mother function $\psi$, and $C_{k}$ is the wavelet coefficients. There is an advantage in requiring orthogonal scaling functions and wavelets. Orthogonal basis functions allow simple calculation of expansion coefficients. The orthogonal complements of $\mathrm{V}_{\mathrm{j}}$ in $\mathrm{V}_{\mathrm{j}+1}$ are defined by $W_{j}$, this means that all members of $\mathrm{V}_{\mathrm{j}}$ are orthogonal to all members of $\mathrm{W}_{\mathrm{j}}[26]$.

$$
\left\langle\varphi_{j, k}(t), \psi_{j, l}(t)\right\rangle=\int \varphi_{j, k}(t) \psi_{j, l}(t) d t=0 \quad j, k, l \in Z
$$

where $\langle$,$\rangle denotes the inner product. So, the wavelet can be represented by a weighted$ sum of shifted scaling function $\varphi(2 \mathrm{t})$ by:

$$
\psi(t)=\sum g(n) \sqrt{2} \varphi(2 t-n) \quad n \in Z
$$

The scaling coefficients are related to the wavelet coefficients by:

$$
g(n)\left(-1^{n}\right) h(1-n)
$$

The function generated by equation (14) gives the prototype or mother wavelet $\psi(x)$ for a class of expansion functions of the form:

$$
\psi_{j, k}(t)=2^{j / 2} \psi\left(2^{j} t-k\right)
$$

The DWT of an image consists of four frequency channels for each level of decomposition. For example, for j-level of decomposition one has:

$W_{\psi}^{D}(j, m, n):$ Coefficients of Diagonal Detail, speckled, (LL)

$W_{\psi}^{V}(j, m, n)$ : Coefficients of Vertical Detail, speckled, (HL)

$W_{\psi}^{H}(j, m, n)$ : Coefficients of Horizontal Detail, speckled, (LH) and

$W_{\varphi}(j, m, n)$ : Coefficients of Approximation $(\mathrm{HH})$. The $W_{\varphi}(j, m, n)$ part at each scale is decomposed recursively, as illustrated in Figure 5.

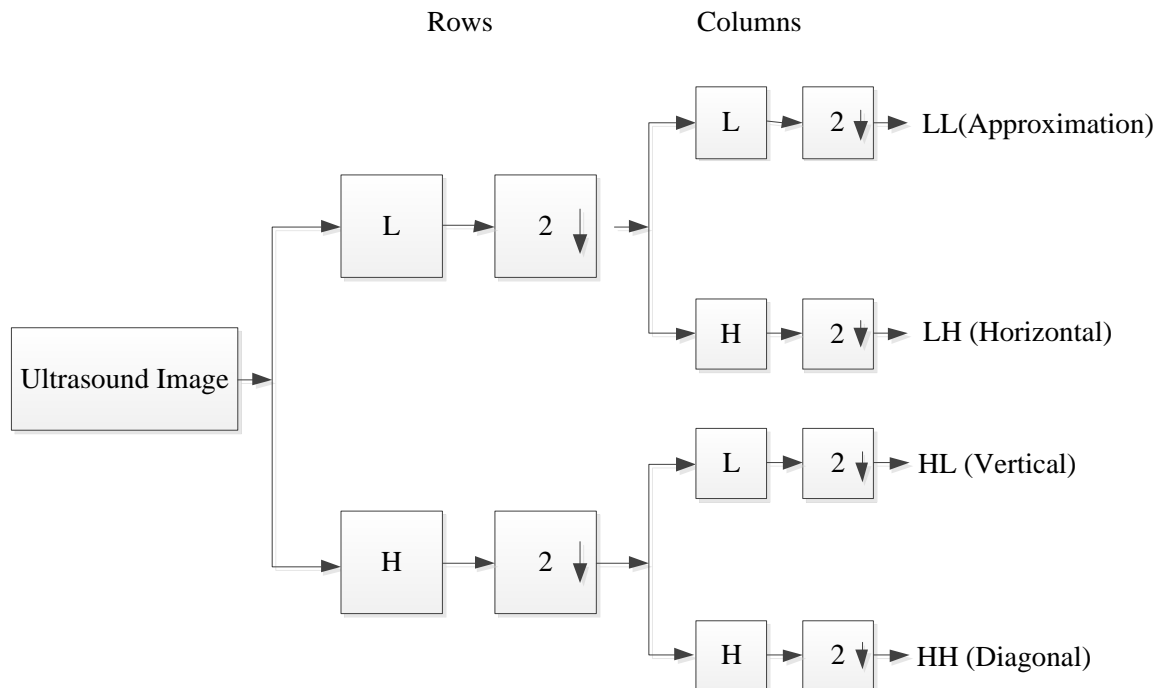

Figure 5. The one level 2D DWT computation. 


\section{Wavelet coefficient quantization}

Before introducing wavelet coefficients extracted from decomposition bands to the stage of run-length matric calculation and then run-length feature extraction, the wavelet coefficients have to be quantized (scaled). The pseudo code below describes this process.

$W_{\max }=\max \left(W_{i j}\right)$, where $\mathrm{i}, \mathrm{j}$ are the depth and width indices of the wavelet coefficients matrix $\mathrm{W}_{\mathrm{ij} \text {. }}$ The quantized coefficients $W_{i j}^{q}$ is describe by the equation (15).

$$
W_{i j}^{q}=\left\lfloor\left(W_{i j}\right)^{2^{n}} / W_{\max }\right\rfloor
$$

$\mathrm{n}=7$ to map each coefficient to the standard gray level ranged from $(0-255)$ which indicates 8-bit / pixel.

\section{Feature extraction}

Feature extraction is the process of obtaining higher-level information of an image such as color, shape and texture. Spectral and textual are two fundamental pattern elements that are interpreted by human. The feature vector used for classification consists initially of five features. These features are Short Run Emphasis, Long Run Emphasis, Run Percentage, Gray Level Non-Uniformity and, Run Length NonUniformity.

\section{Textural features}

While there is no single or formal meaning of the expression "texture", it is utilized to depict the local spatial variations in image brightness, which are subsequently reidentified with properties, for example, coarseness and to properties such as coarseness and regularity [27]. As it was the term "texture" is a descriptor that instinctively gives measures of properties such smoothness, coarseness and consistency. The presented features were derived from Gray Level Run Length Matrix. Hence Galloway proposed the use of a run-length matrix for texture feature extraction.

For a given image, a run-length matrix $p(i, j)$ is defined as the number of runs with pixels of gray level $i$ and run length $j$.

$$
P_{p}(i, j)=p(i, j) \cdot j
$$

For a given image, a run-length matrix $p(i, j)$ is defined as the number of runs with pixels of gray level $i$ and run length $j$. Various texture features can then be derived from this run-length matrix. From the original run-length matrix $p(i, j)$, many numerical texture measures can be computed. The five original features of run length statistics derived by Galloway [28] are as follows.

Short Run Emphasis (SRE):

$$
S R E=\frac{1}{n_{r}} \sum_{i=1}^{M} \sum_{j=1}^{N} \frac{p(i, j)}{j^{2}}=\frac{1}{n_{r}} \sum_{j=1}^{N} \frac{p_{r}(j)}{j^{2}}
$$

Long Run Emphasis (LRE):

$$
L R E=\frac{1}{n_{r}} \sum_{i=1}^{M} \sum_{j=1}^{N} p(i, j) \cdot j^{2}=\frac{1}{n_{r}} \sum_{j=1}^{N} p_{r}(j) \cdot j^{2}
$$


Gray-Level Non-uniformity (GLN):

$$
G L N=\frac{1}{n_{r}} \sum_{i=1}^{M}\left(\sum_{j=1}^{N} p(i, j)\right)^{2}=\frac{1}{n_{r}} \sum_{i=1}^{M} p_{g}(i)^{2}
$$

Run Length Non-uniformity (RLN)

$$
R L N=\frac{1}{n_{r}} \sum_{j=1}^{N}\left(\sum_{i=1}^{M} p(i, j)\right)^{2}=\frac{1}{n_{r}} \sum_{j=1}^{N} p_{r}(i)^{2}
$$

Run Percentage (RP)

$$
R P=\frac{n_{r}}{n_{p}}
$$

In the above, $n_{r}$ is the total number of runs and $n_{p}$ is the number of pixels in the image.

\section{Feature selection}

We used Student's t-test to study if the mean value of a feature is significantly different between the benign and malignant groups. The result of the t-test is the Pvalue, which is compared with a level of significance (a-level). Popular levels of significance are $5 \%(0.05), 1 \%(0.01)$ and $0.1 \%(0.001)$. If the P-value is lower than the $\alpha$-level, it indicates that the feature is powerful enough to be different for the two classes. In this work, ANOVA test is carryout on both two classes which it is a statistical hypothesis test utilizes the variances to check if the means are different. The invalid-hypothesis is rejected when $(P<0.001)$.

\section{Classification}

Support Vector Machine (SVM) which are supervised learning models with associated learning algorithms that analyze data used for classification and regression analysis. An SVM classifier based on a Gaussian radial bases function, which is known to produce hyperplanes for the partition of two datasets, was utilized. All features were standardized before being connected to the classifier. Since the number of nodules, including benignity and malignancy, was generally little compared with the total number of features. SVM uses a linear hyperplane to separate the dataset optimally in a higher dimensional plan when the data is non-linearly related to the input plane [29] and Decision Tree(DT) [30] are to Classifier methods widely used in classification technique. It applies a straight forward idea to solve the classification problem. Decision Tree Classifier poses a series of carefully crafted questions about the attributes of the test record classifier have been employed. 100 thyroid nodule images which consist of 50 benign and 50 malignant ultrasound thyroid images were used. The database used in this work is publicly available database called BETHSEDA system. All thyroid images have been resized to 576 pixels ${ }^{*} 720$ pixels.

\section{Performance measures}

The classification stage is started by generating 10 folds automatically using the MATLAB command CV-partition. These folds are randomly and uniquely select images from the benign and malignant groups but with a constant ratio $(10 \%$ for testing and $90 \%$ for training). The images used each time to train the proposed neural network systems are selected randomly and equally from benign and malignant images groups. 
i.e. $45 \%$ of the images are derived from malignant group and other $45 \%$ are derived from a benign group. The same mechanism is repeated to select images for testing in each fold. The actual accuracy PPV, sensitivity, and specificity can be measured from the average of ten folds.

\section{RESULTS}

Out of 45 features retrieved with the proposed technique (Spectral-Textural), 33 features were selected using P-value, which is the probability of rejecting the null hypothesis assuming that the null hypothesis is true. The P-value has been calculated for all presented features are subjected to the ANOVA (Analysis of Variance between groups) test to obtain the P-value. ANOVA uses variances to decide whether the means are different. Then input features of thyroid nodule images have been fed into the proposed classifiers (SVM and DT). This test uses the variation (variance) within the groups and translates into variation (i.e. differences) between the groups, taking into account how many subjects there are in the groups.

Finally, from the last 45 features, the best 33 were selected which have $P$-value less than or equal to 0.001 . Then the list of features vector members used for the training of classifiers are refers by $\left(^{*}\right)$ in Table 1 below which shows the 45 nominated features from both groups (Benign and Malignant) and show the top 33- features which will form the feature vector. Figure 6 depicts graphical illustration for the nominated features with their $\mathrm{P}$ - value; the numbers indicated in this figure were taken from Table 2(feature number).

The feature vector consists the features which refers by $\left(^{*}\right)$ in the Table 1. Figure 7 depict a graphical illustration for one out of thirty- three features employed in feature vector which passes the ANOVA test and Figure 8 refers to one of those features failed a test. From two figures mentioned, it's noticed that there is large difference in normalized features values ranges which give the classifier better chance in making right decision.

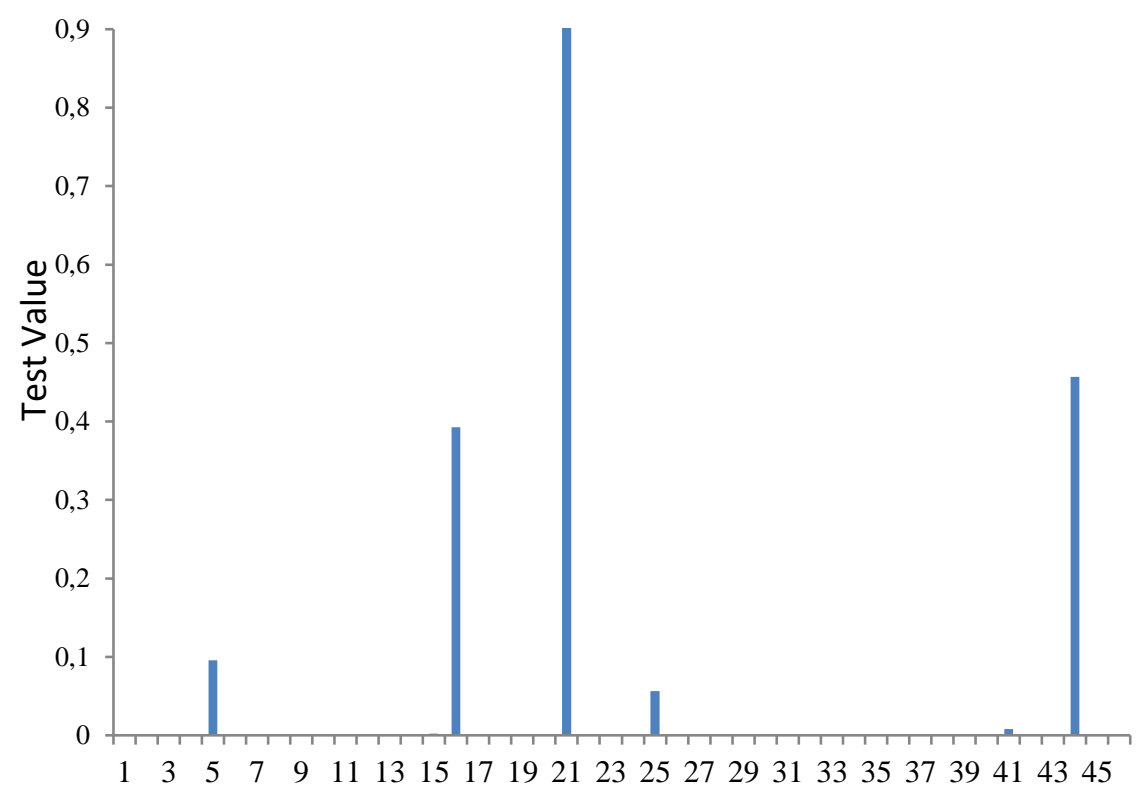

Figure 6. Depicts graphical illustration for the nominated features with their P-value. 
Table 1. shows the 45 nominated features from both groups (Benign and Malignant) and the top 33- features.

\begin{tabular}{|c|c|c|}
\hline Feature name & $\begin{array}{l}\text { Feature } \\
\text { number }\end{array}$ & P-value \\
\hline Short Run Emphasis without wavelet $\left(^{*}\right)$ & 1 & 0.000911513497334381 \\
\hline Long Run Emphasis without wavelet( $\left.{ }^{*}\right)$ & 2 & $1.22146631276738 \mathrm{e}-07$ \\
\hline Run Percentage without wavelet( $\left.{ }^{*}\right)$ & 3 & $1.03640910846964 \mathrm{e}-07$ \\
\hline Gray Level Non-Uniformity without wavelet(*) & 4 & $1.32957362794518 \mathrm{e}-07$ \\
\hline Run Length Non-Uniformity without wavelet & 5 & 0.0957849114029747 \\
\hline Short Run Emphasis with wavelet(LL1) $\left(^{*}\right)$ & 6 & $6.32050844217445 e-25$ \\
\hline Long Run Emphasis with wavelet(LL1) $\left(^{*}\right)$ & 7 & $1.17339934500856 \mathrm{e}-06$ \\
\hline Run Percentage with wavelet(LL1) $\left(^{*}\right)$ & 8 & $4.80724583145179 \mathrm{e}-07$ \\
\hline $\begin{array}{l}\text { Gray Level Non-Uniformity with wavelet(LL1) } \\
\left({ }^{*}\right)\end{array}$ & 9 & $7.82873185155033 e-06$ \\
\hline $\begin{array}{l}\text { Run Length Non-Uniformity with wavelet(LL1) } \\
\left({ }^{*}\right)\end{array}$ & 10 & $1.70303457896002 \mathrm{e}-06$ \\
\hline Short Run Emphasis with wavelet(LH1) $\left(^{*}\right)$ & 11 & $6.20468059151341 e-05$ \\
\hline Long Run Emphasis with wavelet(LH1) $\left(^{*}\right)$ & 12 & $1.11592655445120 \mathrm{e}-09$ \\
\hline Run Percentage with wavelet(LH1) $\left(^{*}\right)$ & 13 & $4.55987278389440 \mathrm{e}-11$ \\
\hline Gray Level Non-Uniformity with wavelet(LH1) & 14 & $\mathrm{NaN}$ \\
\hline Run Length Non-Uniformity with wavelet(LH1) & 15 & 0.00225506533075640 \\
\hline Short Run Emphasis with wavelet(HL1) & 16 & 0.392639619751827 \\
\hline Long Run Emphasis with wavelet(HL1) $\left(^{*}\right)$ & 17 & $9.68304999545235 \mathrm{e}-09$ \\
\hline Run Percentage with wavelet(HL1) $\left(^{*}\right)$ & 18 & $1.24397523691814 \mathrm{e}-09$ \\
\hline Gray Level Non-Uniformity with wavelet(HL1) & 19 & $\mathrm{NaN}$ \\
\hline $\begin{array}{l}\text { Run Length Non-Uniformity with wavelet }(\mathrm{HL} 1) \\
*\end{array}$ & 20 & 2.20567783130527e-07 \\
\hline Short Run Emphasis with wavelet(HH1) & 21 & 0.991540417456596 \\
\hline Long Run Emphasis with wavelet $\left.(\mathrm{HH} 1){ }^{\prime}{ }^{*}\right)$ & 22 & 4.60667951687446e-07 \\
\hline Run Percentage with wavelet $(\mathrm{HH} 1)\left(^{*}\right)$ & 23 & 2.81336696991158e-07 \\
\hline $\begin{array}{l}\text { Gray Level Non-Uniformity with } \\
\text { wavelet }\left({ }^{*}\right)(\mathrm{HH} 1)\end{array}$ & 24 & $2.96443848050847 \mathrm{e}-05$ \\
\hline Run Length Non-Uniformity with wavelet $(\mathrm{HH} 1)$ & 25 & 0.0561987075339243 \\
\hline Short Run Emphasis with wavelet(LL2) $\left(^{*}\right)$ & 26 & $3.00964602938298 e-34$ \\
\hline Long Run Emphasis with wavelet(LL2) $\left(^{*}\right)$ & 27 & $2.92849869326468 \mathrm{e}-05$ \\
\hline Run Percentage with Wavelet(LL2) $\left(^{*}\right)$ & 28 & $9.25137812028412 \mathrm{e}-06$ \\
\hline Gray Level Non-Uniformity with wavelet(LL2) & 29 & $\mathrm{NaN}$ \\
\hline $\begin{array}{l}\text { Run Length Non-Uniformity with } \\
\text { wavelet }\left(\text { LL2 } 2\left(^{*}\right)\right.\end{array}$ & 30 & $1.32147575408594 \mathrm{e}-05$ \\
\hline Short Run Emphasis with wavelet(LH2) $\left(^{*}\right)$ & 31 & $2.08784292943773 e-10$ \\
\hline Long Run Emphasis with wavelet(LH2) $\left(^{*}\right)$ & 32 & $2.94326125263654 \mathrm{e}-10$ \\
\hline Run Percentage with wavelet(LH2) $\left(^{*}\right)$ & 33 & $9.74109588690891 \mathrm{e}-11$ \\
\hline Gray Level Non-Uniformity with wavelet(LH2) & 34 & $\mathrm{NaN}$ \\
\hline $\begin{array}{l}\text { Run Length Non-Uniformity with } \\
\text { wavelet }(\mathrm{LH} 2)\left({ }^{*}\right)\end{array}$ & 35 & 3.32499024991315e-06 \\
\hline Short Run Emphasis with wavelet(HL2) $\left(^{*}\right)$ & 36 & 0.000226553815882494 \\
\hline Long Run Emphasis with Wavelet(HL2) $\left(^{*}\right)$ & 37 & $2.48585306015029 \mathrm{e}-09$ \\
\hline Run Percentage with wavelet(HL2) $\left(^{*}\right)$ & 38 & $2.57349519655975 e-10$ \\
\hline Gray Level Non-Uniformity with wavelet(HL2) & 39 & $\mathrm{NaN}$ \\
\hline $\begin{array}{l}\text { Run Length Non-Uniformity with } \\
\text { wavelet }(\mathrm{HL} 2)\left({ }^{*}\right)\end{array}$ & 40 & $1.85986271977515 \mathrm{e}-06$ \\
\hline Short Run Emphasis with wavelet(HH2) & 41 & 0.00821513769419838 \\
\hline Long Run Emphasis with wavelet( $\mathrm{HH} 2)\left(^{*}\right)$ & 42 & $6.00550576447417 \mathrm{e}-06$ \\
\hline
\end{tabular}




\begin{tabular}{lcl}
\hline \multicolumn{1}{c}{ Feature name } & $\begin{array}{c}\text { Feature } \\
\text { number }\end{array}$ & \multicolumn{1}{c}{ P-value } \\
\hline Run Percentage with wavelet $(\mathrm{HH} 2)\left({ }^{*}\right)$ & 43 & $4.21254452131960 \mathrm{e}-06$ \\
Gray Level Non-Uniformity with wavelet $(\mathrm{HH} 2)$ & 44 & 0.456802524759402 \\
Run Length Non-Uniformity with & 45 & $6.80836848938470 \mathrm{e}-07$ \\
wavelet $(\mathrm{HH} 2)\left({ }^{*}\right)$ & & \\
\hline
\end{tabular}

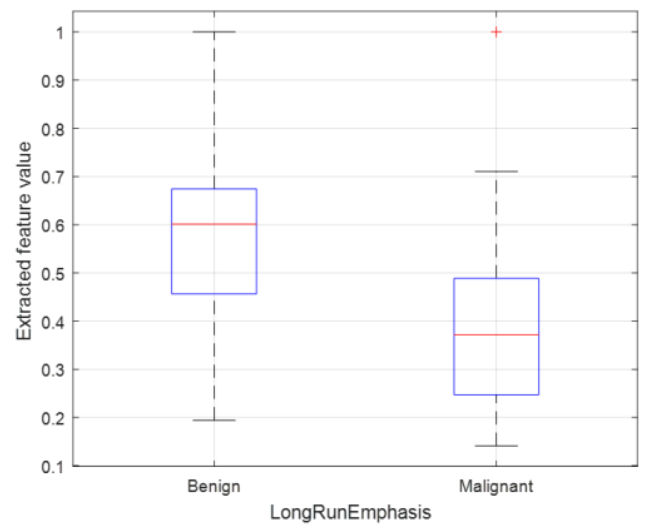

Figure 7. Normalized values for the Difference without wavelet Long Run Emphasis feature for the two categories (Benign and Malignant).

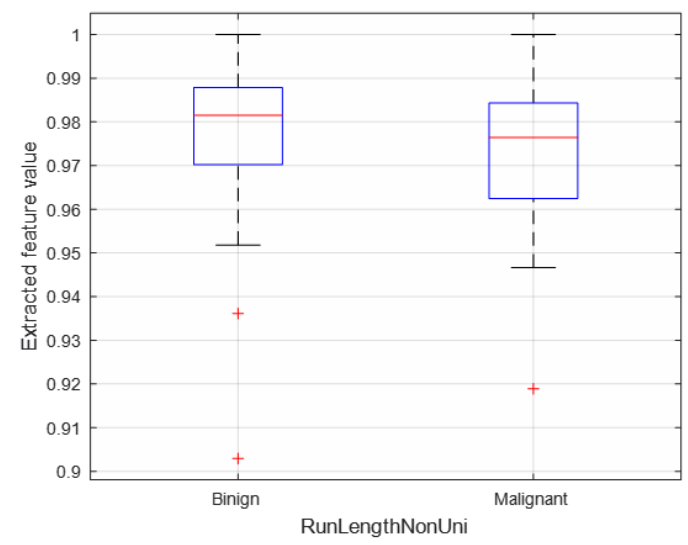

Figure 8. Normalized values for difference without wavelet Run Length Non Unifeature for the two categories (Benign and Malignant) failed the Test

\section{Classification results}

The performance of SVM and DT classifiers are shown in Table 2 using all features extracted from thyroid nodule images and listed in Table 1. Four scenarios have been proposed for thyroid nodule malignancy classification. The first scenario is based on features derived from RLGLM calculated directly from each thyroid image spatial domain and using SVM classifier. The second scenario using the same previous classifier SVM fed by successful features derived from GLRM calculated from image spatial domain features and GLRMs were calculated from 8-band 2-level wavelet decomposition matrices called hybrid features. While in third and fourth scenarios DT has been used as classifier fed by spatial domain features in third scenario and hybrid features in forth scenario. Then 38 features have been selected for this study. Accuracy, positive predictive value (PPV), sensitivity and specificity for all the classifiers using these sets of features. Table 2 shows that for the given dataset the SVM second scenario classifier shows the best classification accuracy of $98 \%$ sensitive of $100 \%$ and specificity of $96 \%$ using 33 ranked features. 
Table 2. Sensitivity, Specificity, Accuracy and PPV values presented by the classifiers using all features for training and testing.

\begin{tabular}{cccccc}
\hline Classifiers & No. of features & Acc (\%) & PPV (\%) & Sn (\%) & Sp (\%) \\
\hline SVM based RLGLM & 5 & 97 & 94 & 94 & 94 \\
SVM based hybrid features & 33 & 98 & 94 & 100 & 94 \\
DT based RLGLM & 5 & 93 & 93.6 & 88 & 94 \\
DT based hybrid RLGLM & 33 & 96 & 96 & 98 & 96 \\
\hline
\end{tabular}

Notations used for: Sn: sensitivity, Sp: specificity, Acc: accuracy, PPV: positive predictive value

The proposed algorithm has the following striking features:

1- From the Table 2. It can be concluded that the average classification accuracy of thyroid nodule class is $96 \%$ and the false positive is zero.

2- The proposed system automatically separates features and uses them with the SVM classifier to predict the class (Benign and Malignant) of the subject. Since no client cooperation is important, the final products are more focused and reproducible contrasted with human interpretations, which may prompt between observer variations. 3-The framework is created utilizing ten times cross approval information resampling, subsequently, it can predict the obscure class all the more precisely. So the proposed strategy is stronger.

4- The framework can consequently recognize all the unusual subjects as strange (no false positive cases), as the sensitivity of the framework is $100 \%$. This will decrease the work heap of clinicians a great deal, as they have to concentrate just on the typical classes.

5- The accuracy of this framework can be expanded further utilizing huge database with various images, other non-linear features, and better classifiers.

6 - Wavelet features can catch the unobtrusive varieties in the pixels easily and are more powerful to noise.

The Receiver Operating Curve(ROC) is an objective means for accessing the performance of an imaging system. For comparisons of performance, we applied ROC curves for the proposed scenarios based on SVM and DT. The area under the curve denotes the accuracy of third and four scenarios based on DT illustrated in Figures 9,10, first and second scenarios based on SVM are shown in Figures 11,12.

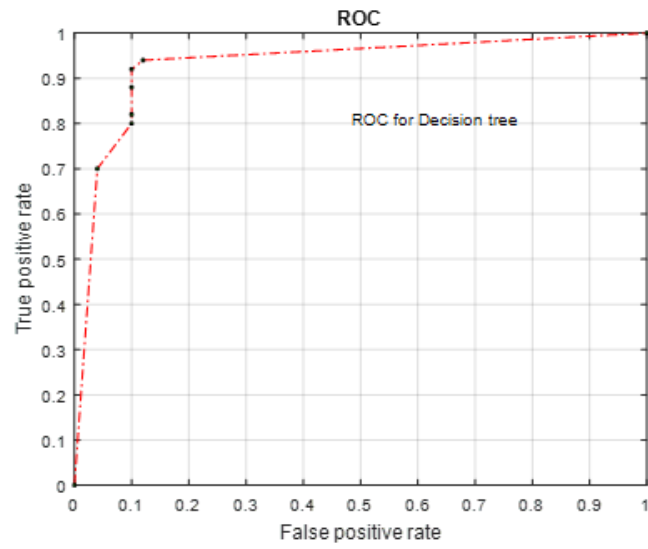

Figure 9. Receiver Operating Curve of spatial domain (DT).

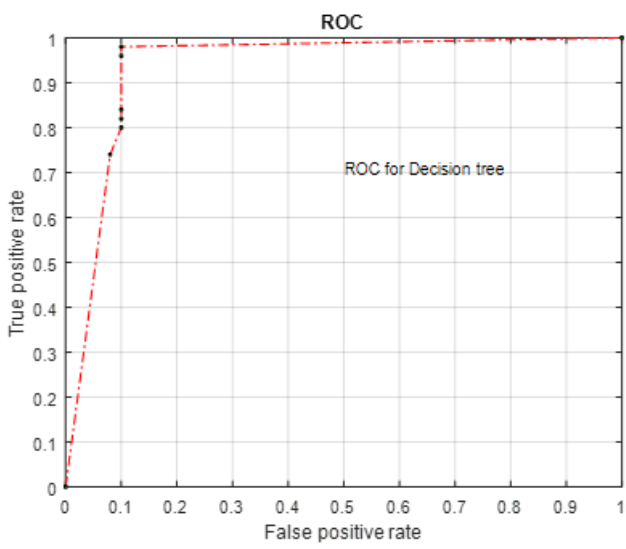

Figure 10. Receiver Operating Curve of (DT + hybrid). 


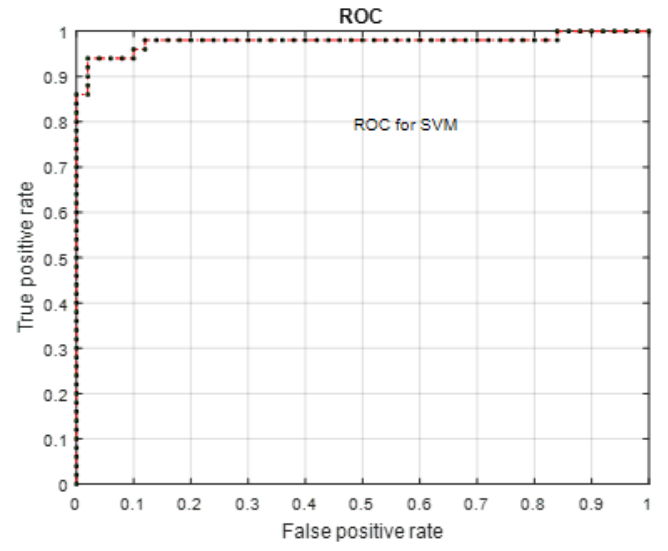

Figure 11. Receiver Operating Curve of spatial domain (SVM).

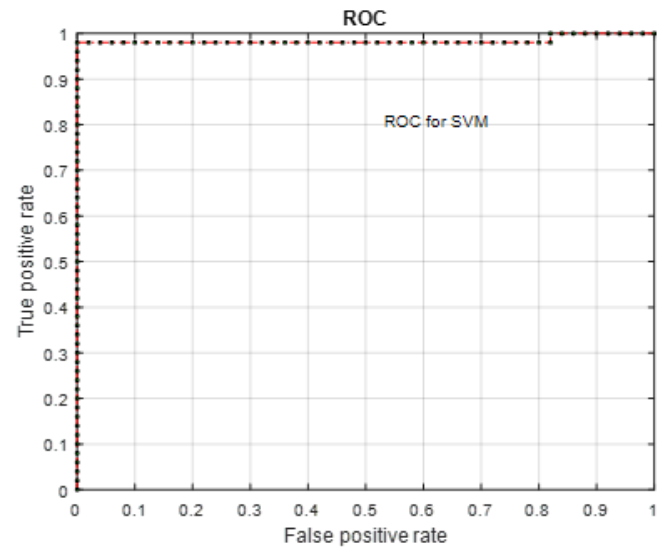

Figure 12. Receiver Operating Curve of (SVM+hybrid).

\section{CONCLUSION AND FUTURE WORK}

Nodular thyroid disease is common and because of the associated risk of malignancy and hyper-function, these nodules have to be examined thoroughly. The early identification of thyroid cancer may avoid the surgery operations. This method is efficient in detecting and classifying the thyroid nodules in ultrasound images for the proposed dataset. GLRLM based on wavelet decomposition (hybrid) features are used to detect the early stage of thyroid nodule cancers. The thyroid nodule images used in this work are obtained from the publicly available TI-RADS databases. In this work, four scenarios have been proposed based on two classifier DT and SVM whose quantitative analysis is done by calculating the accuracy, sensitivity, and specificity. The best classification accuracy obtained is $98 \%$ from fourth scenario SVM classifier and compared to the featured derive for GLRLM matrices calculated from spatial domain.

$\mathrm{ROC}$ analysis for nodules classification as far as ranges under curves additionally demonstrated a comparative execution in results for both $C A D$ and visual review by radiologists. Accordingly, the proposed CAD framework, which produces reproducible and quantitative results, can be the advantage as the alternative choice for radiologists by giving data on benignity and malignancy of a thyroid lesion in a self-loader way on US images, and consequently can decrease heavy burden due to the increasing number of incidents. Later on, enhancing the proposed CAD framework may, in the end prompt an economically accessible thyroid framework for clinical applications. In future work, the curvelet transform based statistical features must be studied and compared with the current system. Also the debauching wavelet transform might be considered at future to replace the current Haar wavelet.

Conflicts of Interest: The authors declare no conflict of interest.

\section{REFERENCES}

1. Siegel RL, Miller KD, Jemal A. Cancer statistics. CA Cancer J. Clin. 2018;68(1):7-30. doi:wiley.com/10.3322/caac.21442.

2. Edge SB, Compton CC. The american joint committee on cancer: The 7th edition of the AJCC cancer staging manual and the future of TNM. Ann Surg Oncol. 2010;17:1471-4.

3. Keramidas EG, lakovidis DK, Maroulis D, Karkanis S. Efficient and Effective Ultrasound Image Analysis Scheme for Thyroid Nodule Detection. Kamel M, Campilho A, editors. Image Analysis and Recognition. ICIAR 2007; 1052-60. Lecture Notes in Computer Science,. Springer, Berlin, Heidelberg. doi: 10.1007/978-3-540-74260-9_93.

4. Doherty GM, Haugen BR, Mazzaferri EL, Mciver B, Ph D, Pacini F, et al. Revised American 
Thyroid association management guidelines for patients with thyroid nodules and differentiated. Thyroid Cancer. 2009;19(11).

5. Chow LS, Gharib H, Goellner JR. Nondiagnostic thyroid fine-needle aspiration cytology: management dilemmas. Thyroid. 2001;11(12).

6. Feld S. AACE clinical practice gudelines for the diagnosis and munagement of thyroid nodules. Thyroid Nodule Task Force. Endocr Pract. 1996;2:78-84.

7. Soto GD, Halperin I, Squarcia M, Lomeña F, Domingo MP. Update in thyroid imaging. the expanding world of thyroid imaging and its translation to clinical practice. Hormones. 2010; 9:287-98.

8. Zhou S, Shi J, Zhu J, Cai Y. Wang R. Biomedical signal processing and control shearletbased texture feature extraction for classification of breast tumor in ultrasound image. Biomed Signal Process Control. 2013;8:688-96. doi:org/10.1016/j.bspc.2013.06.011.

9. Gao L, Liu R, Jiang Y, Song W, Wang Y, Liu J, et al. Computer-aided system for diagnosing thyroid nodules on ultrasound: a comparison with radiologist-based clinical assessments. Head Neck. 2018;40:778-83.

10. Nyúl L. Retinal image analysis for automated glaucoma risk evaluation. In: Proceedings of SPIE-The International Society for Optical Engineering. 2009;7497:74971C. doi:org/10.1117/12.851179.

11. Cheng HD, Shan J, Ju W, Guo Y, Zhang L. Automated breast cancer detection and classification using ultrasound images: a survey. Pattern Recognit. 2010;43:299-317. doi: org /10.1016/j.patcog.2009.05.012.

12. Xu G, Wang Sh, Yang T, Jiang W. A new neutrosophic approach based on TOPSIS method to image segmentation. IJCCC. 2018;13:1047-61. doi: 10. 15837/ ijccc . 2018. 6. 3268.

13. Acharya UR, Sree SV, Krishnan MM, Molinari F, Saba L, Ho SYS, et al. Atherosclerotic risk stratification strategy for carotid arteries using texture-based features. Ultrasound Med Biol. 2012;38:899-915. doi:10.1016/j.ultrasmedbio.2012.01.015. Epub 2012 Apr 21.

14. Moon WK, Shen YW, Huang CS, Chiang LR, Chang RF. Original contribution computeraided diagnosis for the classification of breast masses in automated whole in breast ultrasound images. Ultrasound Med Biol. 2011;37:539-48. doi:10.1016/j.ultrasmedbio.2011.01.00.

15. Jenitta A, Ravindran RS. Content based geographic image retrieval using local vector pattern. Braz Arch Biol Technol. 2018;61:1-10.

16. Tang $X$. Texture information in run-length matrices. IEEE Trans Image Process. 1998;7:1602-9.

17. Conners RW, Harlow CA. A theoretical comparison of texture algorithms. IEEE Trans Pattern Anal Mach Intell. 1980;2:204-22.

18. Chu A, Sehgal CM, Greenleaf JF. Use of gray value distribution of run lengths for texture analysis. Pattern Recognition Letters 1990; 11:415-20.

19. Bhuvaneswari NR, Sivakumar VG. Novel image classification technique using particle filter framework optimised by multikernel sparse representation. Braz Arch Biol Technol. 2016; $59: 1-8$.

20. Rose RR.; Meena K.; Suruliandi A. An empirical evaluation of the local texture description framework-based modified local directional number pattern with various classifiers for face recognition. Braz Arch Biol Technol 2016;59:1-17.

21. Khaw PT. Glaucoma: a colour manual of diagnosis and treatment. British Journal of Ophthalmology. 1998;82:97-100.

22. Van de Wouwer G.; Scheunders P.; Van Dyck D. Statistical texture characterization from discrete wavelet representations. IEEE Transactions on Image Processing. 1999;8:592-8. doi:10.1109/83.753747.

23. Kwak JY.; Jung I.; Baek JH.; Baek SM.; Choi N.; Choi YJ, et al. Image reporting and characterization system for ultrasound features of thyroid nodules: Multicentric Korean retrospective study. Korean J Radiol. 2013;14:110-7. 
24. Sudarshan VK.; Rama M.; Mookiah K.; Acharya UR.; Chandran V.; Molinari F, et al. Application of wavelet techniques for cancer diagnosis using ultrasound images : a review. Comput Biol Med. 2016;69:97-111. doi:org/10.1016/j.compbiomed.2015.12.006.

25. Charalampidis D.; Kasparis T. Wavelet-based rotational invariant roughness features for texture classification and segmentation. IEEE Trans Image Process. 2002;11:825-37.

26. Tourassi GD. Journey toward computer-aided diagnosis: role of image texture analysis. Radiology. 1999;213:317-20. doi: /10.1148/radiology.213.2.r99nv49317.

27. Gonzalez RC.; Woods RE. Digital image processing 2nd ed. Nueva Jersey; 1987. p. 793.

28. Mary M. Texture analysis using gray level run lengths. Computer Graphics and Image Processing 1975; 4; 172-179.

29. Mukherjee S, Tamayo P, Slonim D, Verri A, Golub T, Mesirov JP, et al. Support Vector Machine Classification of Microarray Data. Massachusetts Institute of Technology. 1998. Technical Report CBCL. No. 182. Available from: http://www.ai.mit.edu.

30. Barros RC, Freitas AA. Automatic design of Decision-Tree induction algorithms. SpringerBriefs in Computer Science 2015;176:18. doi: 10.1007/978-3-319-14231-9.

(c) 2018 by the authors. Submitted for possible open access publication under the terms and conditions of the Creative Commons Attribution (CC BY NC) license (https://creativecommons.org/licenses/by-nc/4.0/). 